\title{
A Novel Orthomyxovirus of Ducks - Ultrastructural Similarities and Differences to Arenaviruses
}

\author{
A. Hyatt, S.Crameri, A. Leis ${ }^{1}$, C. Holmes, I. Smith, S. Shan, and G Marsh \\ AAHL Biosecurity Microscopy Facility CSIRO, Livestock Industries, Australian Animal Health \\ Laboratory, Private Bag 24 Geelong, Vic 3220
}

Samples from farmed diseased Muscovy ducks, Cairina moschata, (Australia) were submitted for investigation. The disease was attributed to Salmonellosis. However routine virological examination of the samples revealed the presence of a CPE (cytopathic effect) causing agent.

The samples were analyzed by PCR for avian influenza virus (AIV), Newcastle disease virus (NDV), duck virus hepatitis (DVH), avian paramyxovirus (APMV) and Duck enteritis virus (DEV); all assays returned negative results. Examination of the infected cell cultures and associated supernatant by transmission electron microscopy revealed the presence of a virus that was absent from control (non-infected) cells. The viruses budded from the plasma membrane, had prominent surface projections and approximated $100 \mathrm{~nm}$ in diameter. Internally ribonucleoprotein complexes (RNPs) were present together with electron dense granular particles. This data set was consistent with the virus not being a paramyxovirus, enterovirus or a herpesvirus. The ultrastructure shared characteristics with viruses from the families Orthomyxoviridae and Arenaviridae.

To confirm the identity of the virus, samples were analyzed by 454 sequencing. The sequence revealed the virus to be a member of a novel genus of the family Orthomyxoviridae. Members of this genus include Quaranfil virus (QRFV), Johnston Atoll virus (JAV) and Lake Chad virus (LKCV), [1]. Until recently, conventional approaches to characterize QRFV, JAV, and LKCV did not result in a definitive classification of these viruses. Examination by electron microscopy suggested the viruses belonged to the arenavirus family [2].

Ultrastructural identification of this group of viruses is challenging. We present data derived from a variety of techniques to facilitate further accurate identification and differentiation of these viruses from classical orthomyxoviruses and arenaviruses. The techniques include negative contrast electron microscopy and examination of sections via classical transmission electron microscopy and tomography.

\section{References}

[1] R.M. Presti et al., J. Virol. 83 (2009) 11599-11606.

[2] H.G. Zeller et al., Arch. Virol. 108 (1989) 191-209.

[3] C. Messaoudii et al., BMC Bioinformat. 8 (2007) 288.

[4] C.O.S. Sorzano et al., BMC Bioinformat. 10 (2009) 124. 


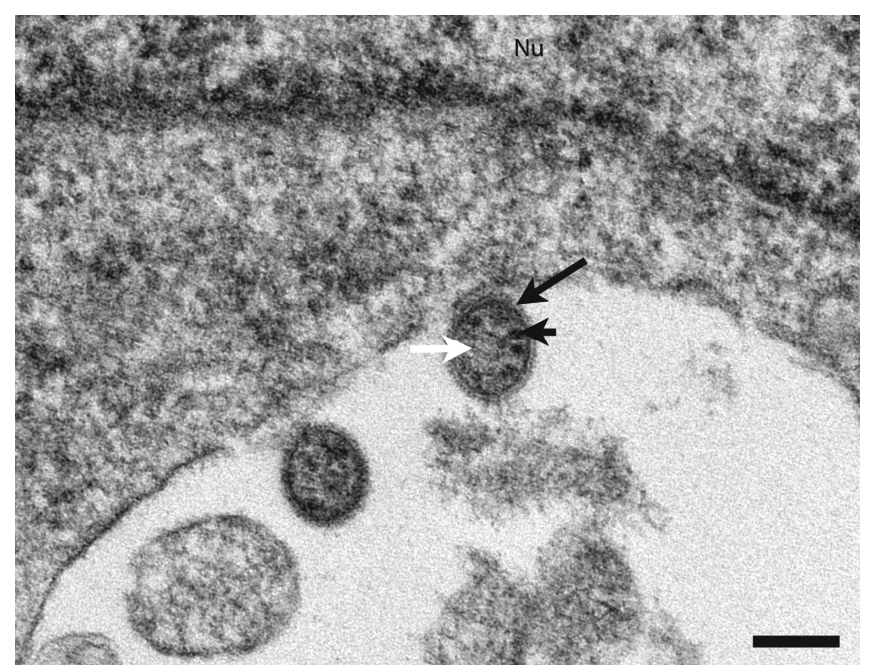

FIG. 1. Transmission electron micrograph of a negative stained virus. Arrow, surface projections. Bar represents 50nm.

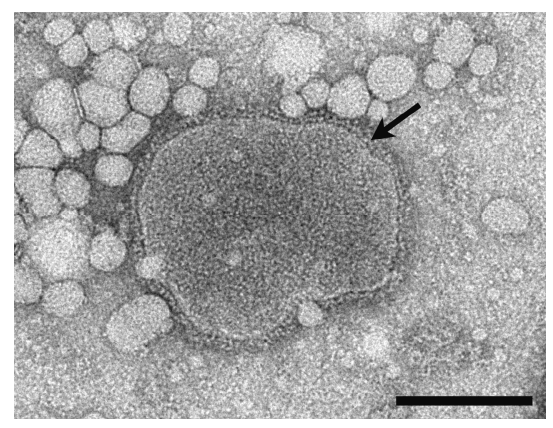

FIG.2. Transmission electron micrograph of an ultrathin section of an infected cell. $\mathrm{Nu}$, nucleus; large arrow surface projections, small arrow, electron dense granular particle; white arrow, RNP. Bar represents 100nm.

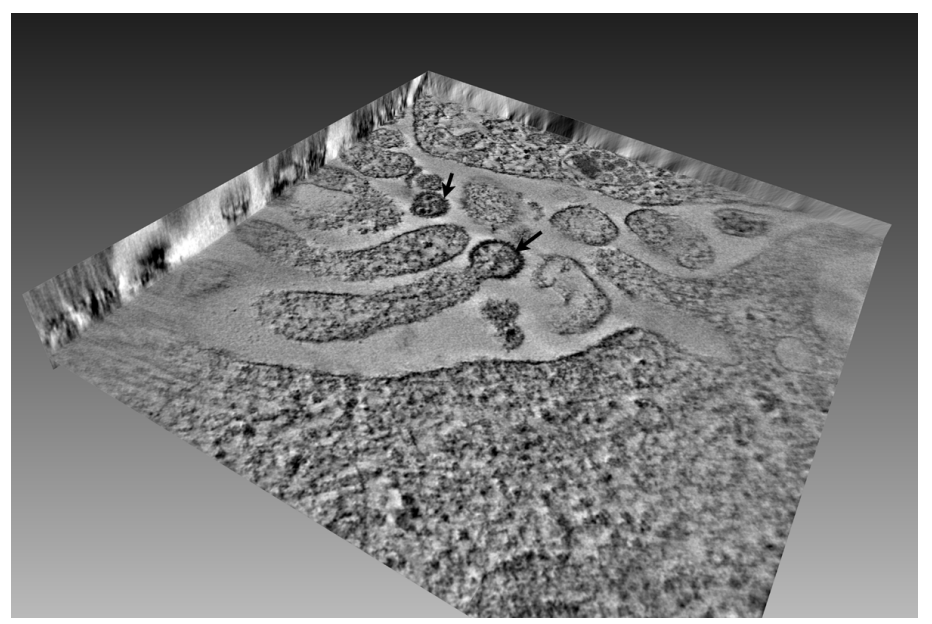

FIG.3. Section of a virus-infected cell from a tomogram derived from a 90nm epoxyembedded and stained section. 